\title{
¿Qué caracterizan las elecciones al Parlamento Europeo? Estudio de la estrategia electoral para las elecciones al PE entre 2004 y 2019: tipología de candidatos y temáticas de campaña
}

\author{
What characterize the elections to the European Parliament? Electoral \\ strategy study for the EP elections between 2004 and 2019: type of \\ candidates and campaign topics
}

\begin{abstract}
Caballero-Escusol, Adrián
Universitat de Vic - Universitat Central de Catalunya (UVic-UCC) adrian.caballero@uvic.cat
\end{abstract}

Ginesta, Xavier Universitat de Vic - Universitat Central de Catalunya (UVic-UCC) xavier.ginesta@uvic.cat

San Eugenio, Jordi de Universitat de Vic - Universitat Central de Catalunya (UVic-UCC) jordi.saneugenio@uvic.cat

Forma de citar este artículo:

Caballero-Escusol, A., Ginesta, X. y San Eugenio, J. de (2021).¿Qué caracterizan las elecciones al Parlamento Europeo? Estudio de la estrategia electoral para las elecciones al PE entre 2004 y 2019: tipología de candidatos y temáticas de campaña. RAEIC, Revista de la Asociación Española de Investigación de la Comunicación, 8(16), 313-336. https://doi.org/10.24137/raeic.8.16.15 


\section{Resumen:}

El presente artículo presenta un estudio cuantitativo cuyo principal objetivo es determinar las características de una campaña electoral al Parlamento Europeo, específicamente aquellas vinculadas al emisor de los mensajes políticos: tipología de candidatos y temas de campaña. Consideradas como elecciones de segundo orden, la literatura las ha caracterizado de manera particular respecto a elecciones nacionales y regionales y el presente artículo servirá como evidencia empírica de cuáles son las características que se le pueden atribuir a la estrategia política y de comunicación electoral de los comicios al Parlamento Europeo. Con dicho objetivo en mente, se han analizado las características de los actos de campaña (edad, ámbito y sexo de los protagonistas, temáticas tratadas en dichos actos, etc.) de los partidos políticos de España y Francia que, en algún momento entre 2004 y 2019, han obtenido representación en el Parlamento Europeo. Entre las principales conclusiones encontraremos que la agenda mediática europea queda en un segundo plano, que la media de edad de los cabezas de lista supera casi siempre los 50 años y que, aunque aún la brecha es amplia, entre 2004 y 2019 la presencia de mujeres como cabezas de cartel se ha incrementado.

Palabras clave: elecciones al Parlamento Europeo, elecciones de segundo orden, comunicación electoral, agenda mediática

\section{Abstract:}

This article presents a quantitative study whose main goal is to determine the characteristics of an electoral campaign for the European Parliament, specifically those related to the issuer: typology of candidates and topics of campaign. Considered as second-order elections, the literature has characterized them in a particular way with respect to national and regional elections and this article will serve as empirical evidence of the characteristics that can be attributed to the political communication of the elections to the European Parliament. With this objective in mind, the characteristics of the campaign events have been analysed (age, scope and gender of the protagonists, 
themes dealt with in said events, etc.) of the political parties in Spain and France that, sometime between 2004 and 2019, they have obtained representation in the European Parliament. Among the main conclusions we will find that the European media agenda remains in the background, that the average age of the heads of the list almost always exceeds 50 years of age and that, although the gap is still wide, between 2004 and 2019 the presence female headliners has increased.

Keywords: European Parliament elections, second order elections, media agenda, electoral communication

\section{INTRODUCCIÓN}

El objeto de estudio del presente artículo son las campañas electorales al Parlamento Europeo. Más concretamente, la comunicación política y la estrategia de los partidos políticos a la hora de preparar las campañas electorales para maximizar votos en las elecciones al Parlamento Europeo, las únicas supranacionales que se celebran en todos los Estados miembro de la Unión Europea y que, por sus características, son singulares y diferentes a las elecciones generales nacionales, pero también las regionales y locales en la mayoría de dichos Estados miembro.

Esa singularidad es la que hace interesante analizar qué características tienen las campañas electorales en estas elecciones al Parlamento Europeo, centrándonos específicamente en aquellos aspectos endógenos tanto de la elección del candidato (por su perfil: edad, sexo, ámbito político), como de aquellos propios de una estrategia de campaña (como, por ejemplo, la agenda de la campaña, los temas y los ámbito de la misma).

El trabajo se justifica en tanto la literatura ha clasificado las elecciones al Parlamento Europeo como elecciones de segundo orden, un concepto original de Reif y Schmitt (1980), y que dota a los comicios de unas características especiales que se definirán con exactitud en la sección de marco teórico. Algunas de estas características han sido 
analizadas en el presente estudio para establecer conclusiones sobre si, hoy en día, las elecciones al Parlamento Europeo continúan siendo elecciones de segundo orden.

\subsection{OBJETIVOS}

El presente artículo tiene dos objetivos. Por un lado, se tratará de determinar si la agenda temática de las campañas electorales al Parlamento Europeo es efectivamente europea o, como marca la literatura, resulta ser nacional, con cuestiones relevantes a nivel regional o nacional, desplazando los temas de implicación y relevancia europea a un segundo plano.

Por otro lado, el segundo objetivo es determinar qué factores caracterizan la estrategia política y de comunicación en una campaña electoral al Parlamento Europeo, centrándonos principalmente en el rol del emisor (el candidato y su agenda mediática de campaña). La principal aportación del presente texto es un estudio cuantitativo en el que se han analizado diferentes factores de la comunicación electoral y su uso por parte de los diferentes partidos políticos de España y Francia que, al menos una vez, obtuvieron representación en el Parlamento Europeo en las cuatro elecciones europeas celebradas entre 2004 y 2019.

\section{MARCO TEÓRICO Y CONTEXTUAL}

\subsection{COMUNICACIÓN POLÍTICA Y ELECTORAL}

Un concepto central en el presente artículo es el de la comunicación política, tratado largamente por una literatura en la que podemos encontrar diferentes propuestas de definición. Pioneros en este campo, como Lasswell o Edelman, entre otros, bebían de diferentes disciplinas como la sociología, el periodismo, las relaciones públicas e incluso la antropología, a partir de las cuales se empezó a construir el concepto de comunicación política (Bennet e lyengar, 2008). Aunque algunos autores, como Mazzoleni (2010), argumentan que la historia de la comunicación política se remonta a tiempos de la república romana, para multitud de autores el rápido desarrollo de la disciplina se dio a mediados del siglo XX (Canel, 2006). Es entonces cuando diferentes autores tratan de 
definir la disciplina, destacando Denton y Woodward (1998), que asimilan como comunicación política:

a) Toda forma de comunicación llevada a cabo por políticos o actores políticos con el objeto de conseguir objetivos específicos

b) Toda comunicación dirigida a estos actores por parte de acortes no-políticos, como votantes o columnistas

c) Toda comunicación sobre actores políticos y sus actividades, como las noticias, editoriales, y toda discusión mediática

Desde la aparición de los medios de comunicación se considera que la comunicación política toma cuerpo como disciplina. Aunque muchos autores apuntan a la televisión como el formato que la impulso, algunos, como Toni Aira (2008), apuntan a la campaña de las elecciones presidenciales de Estados Unidos de 1952, cuando ambos candidatos, Eisenhower y Stevenson, reservaron, por primera vez, una partida presupuestaria para la comunicación política (Aira, 2008). No obstante, tanto Aira como otros autores señalan otra campaña presidencial estadounidense, la que enfrentó en 1960 a J. F. Kennedy con Richard Nixon, como el inicio de la comunicación política moderna (Maarek, 2011).

Con la llegada de Internet nacen nuevos medios de comunicación nativos digitales y, en global, el número de medios se multiplica, con lo que la atención que reciben los líderes políticos también lo hace. Así, a partir de finales de los años 90 del siglo XX y ya en el siglo XXI surge la preocupación entre los diferentes actores políticos de profesionalizar la comunicación política, apareciendo una nueva figura de asesor y experto en comunicación política, el spin doctor (Aira, 2008).

Con un equipo de comunicación cada vez ocupando más espacio de poder en las instituciones y los partidos políticos, se crea cada vez más contenido y los medios de comunicación, necesitados de dicho contenido para ganar más y más audiencia en un contexto de crisis del sector a inicios del siglo XXI se inclinan por un tratamiento de la información que mezcla la información con el entretenimiento (infotainment), tratando 
de retener la atención de la audiencia con tertulias, debates y otros tipos de contenidos (Mazzoleni, 2010).

Así, los partidos políticos entran en competencia por situar sus mensajes en los cada vez mayores espacios que les ofrecen los medios de comunicación. En este contexto entran en juego las redes sociales, como Facebook o Twitter, nacidas a mediados de la primera década del siglo XXI, que se caracterizan por el consumo de mensajes y contenidos cortos. Así, autores como Canel (2006) califica de "periodismo de titulares" el tipo de periodismo político del siglo XXI. Conscientes de ello, los profesionales de la Comunicación Política trabajan la comunicación del día a día, así como la de campaña electoral con una estrategia orientada a audiencias específicas y con mensajes cortos para satisfacer dichas audiencias. Algunos autores ya alertan que esta entrada de una visión tan comercial, propia del marketing, impide una sociedad informada y crítica (Mazzoleni, 2010).

Este concepto, el marketing político, es desde finales del siglo XX parte integral de la comunicación política, pero especialmente de las campañas electorales (Martínez Pandiani, 2004). Para autores como Philippe Maarek (2011, p. 31), el marketing político es la "política genuina de la comunicación política, una estrategia global que involucra el diseño, la racionalización y la transmisión de la comunicación política". Sobre las posibles diferencias entre el marketing político en una campaña electoral y el marketing comercial, este mismo autor establece tres diferencias:

a) El 'mercado' en un contexto electoral es temporal y se da cada cierto tiempo; el 'mercado' en el contexto comercial es un continuo

b) Por cada contienda electoral, la organización es nueva, mientras que en el contexto comercial es estable

c) En el marketing político electoral, lo importante es el número relativo (sacar el mayor porcentaje de voto); en el comercial lo que importa es el número absoluto

Con todo, el marketing político y electoral se ha abierto paso en los últimos años, especialmente con la universalización del uso de las nuevas tecnologías, como Internet. 
Si bien se recordará al candidato demócrata en primarias de 2004, Howard Dean, como uno de los primeros políticos en hacer uso intensivo de vídeos, blog y correo electrónico, quien convierte las nuevas tecnologías en una forma en sí misma de hacer política y ganar contiendas electorales es el expresidente de los Estados Unidos, Barack Obama (Castro, 2012). Es uno de los primeros grandes ejemplos de campaña multicanal, empleando las herramientas digitales en su campaña de 2008 para captar hasta 13 millones de correos electrónicos y cinco millones de seguidores en redes sociales. Su comunicación pasó de unidireccional a fomentar la participación activa de dichos seguidores y, para no pocos autores, este marketing político digital fue clave en su éxito en la carrera a la Casa Blanca (véase Ramentol, 2008 y Castro, 2012).

En los últimos años, además, las propias campañas electorales han cobrado mayor relevancia. La "mediatización" (López-García et al., 2018) de la política se entiende mejor si se observa la atención que reciben estas campañas electorales, donde el foco de los investigadores se pone en factores como los analizados en este trabajo, como los temas que ponen sobre la mesa los partidos políticos (López-García et al., 2018)

\subsection{LA POLÍTICA EN EL PARLAMENTO EUROPEO}

El Parlamento es la institución de la Unión Europea que representa a sus ciudadanos. Desde 1979, se celebran elecciones generales a este Parlamento Europeo una vez cada cinco años. Hasta esta fecha, el Parlamento (o Asamblea en tiempos anteriores) ya existía, aunque su conformación se hacía con diputados designados por los parlamentos nacionales de los diferentes Estados miembro (Morata, 1998, p. 28).

El Parlamento Europeo, que siempre se ha caracterizado por una participación relativamente baja en los sucesivos comicios, empezó a cobrar relevancia dentro del proceso legislativo europeo a partir de 1993, con el Tratado de Maastricht por el cual se establecía formalmente la Unión Europea y se introducía el llamado método de la codecisión, que igualaba sus poderes competenciales a los del Consejo de la Unión Europea (Auel y Rittberger, 2006). Aunque esto no frenó la baja participación electoral, con los años el Parlamento Europeo ha adquirido más peso en el proceso legislativo, 
convirtiéndose en una de las tres principales instituciones en la toma de decisiones, junto con el mencionado Consejo de la UE y la Comisión Europea (Schmitt y Teperoglou, 2015).

En este contexto de baja participación y desconocimiento por parte de la población, el Parlamento Europeo se ha esforzado en los últimos años en llegar a la población, invirtiendo en una comunicación institucional masiva sobre las competencias y utilidad de la institución. Dicha comunicación institucional se suma a los esfuerzos globales de las principales instituciones de la UE, como la Comisión Europea o el Consejo, quienes también hacen ingentes esfuerzos comunicativos. Para algunos autores, estas estrategias "han convertido a la Unión Europea en el departamento de comunicación institucional más grande del mundo" (Olmo López y Navarro Moreno, 2014, p. 495).

Por lo que respecta a las elecciones al Parlamento Europeo y su funcionamiento, éstas se celebran una vez cada cinco años en todos los Estados miembro a la vez ${ }^{1}$. La circunscripción electoral en los comicios al Parlamento Europeo es el Estado miembro. Así, las listas electorales se definen país por país, con lo que los ciudadanos españoles votan listas de partidos y coaliciones españolas, diferentes de las listas y coaliciones que se presentan en otros Estados miembro.

Tras la salida del Reino Unido como Estado miembro, el Parlamento Europeo está compuesto por 705 eurodiputados, que se eligen de manera proporcional por cada circunscripción electoral (Estado miembro) en función de su población. En la práctica, Alemania es el Estado que más eurodiputados aporta, con 96, seguida por Francia (79) e Italia (76). España es el cuatro Estado miembro con mayor representación (59 eurodiputados). Los países que menor número de eurodiputados escogen son Chipre, Luxemburgo y Malta, con seis cada uno.

Una vez escogidos, los eurodiputados se agrupan en el Parlamento Europeo según su afiliación política. Los diferentes partidos políticos de cada país están representados por partidos políticos europeos que, a su vez, en el Parlamento Europeo se alían para

\footnotetext{
${ }^{1}$ En algunos Estados miembro, los comicios pueden durar todo un fin de semana o celebrarse en sábado en lugar de domingo, como ocurre en la mayoría de los países. Pero, en definitiva, las elecciones se celebran en los mismos días en todos los Estados miembro a la vez.
} 
conformar grupos parlamentarios. Así, se entiende que existen más partidos que grupos parlamentarios y que éstos últimos varían casi en cada legislatura en el Parlamento Europeo.

\subsection{ELECCIONES DE SEGUNDO ORDEN}

Los comicios al Parlamento Europeo son considerados por la literatura como elecciones de segundo orden. A pesar de que no son los únicos etiquetados como tal, Reif y Schmitt (1980) utilizan las primeras elecciones al Parlamento Europeo, en 1979, para ejemplificar el concepto de second order election, llegando a la conclusión de que las elecciones al Parlamento Europeo son elecciones nacionales simultáneas. Esto es: disputas electorales donde la campaña y la agenda mediática está marcada por temas de interés nacional, no europeo.

La principal aportación teórica de Reif y Schmitt, sin embargo, fue atribuir una serie de características a las elecciones de segundo orden. Dichas características, con el paso de los años, han sido analizadas y comprobadas por diferentes autores y, a pesar de matices introducidos por otros autores, no han perdido sentido y relevancia. Para Reif y Schmitt (1980), en definitiva, las elecciones de segundo orden comparten cuatro características principales:
a) Menor participación que en las elecciones generales nacionales
b) Incremento de votos para partidos pequeños y de nueva creación
c) Incremento en el número de votos nulos
d) Los partidos del gobierno tienden a perder votos

Esta aportación ha sido refrendada por estudios posteriores, como el de Norris y Reif (1997), que, partiendo del trabajo de Reif y Schmitt (1980), analizan cuatro convocatorias electorales al Parlamento Europeo para confirmar buena parte de las afirmaciones que 17 años antes hicieron los autores originales. Más tarde, en 2007, Hix y Marsh analizaron las seis convocatorias electorales al Parlamento Europeo que se habían producido desde 1979 para señalar que hasta un 40\% de volatilidad en el voto en este tipo de elecciones se debía a un trasvase de voto de los partidos en el gobierno 
nacional hacia pequeños partidos, confirmando así la característica b) antes mencionada. Por último, Hobolt et al. (2009) siguen la línea explicativa de Hix y Marsh (sobre la pérdida de voto de los partidos en el gobierno), añadiendo el factor del euroescepticismo, apuntando a que precisamente, al tratarse de unas elecciones de segundo orden, los ciudadanos europeos optan por partidos más cercanos a su ideología y su visión más euroescéptica. "Los partidos en el gobierno son, por norma general, bastante más proeuropeos que el votante tipo", llegan a afirmar en su estudio estos autores (Hobolt et al., 2009, p. 93).

Si nos centramos en las elecciones al Parlamento Europeo, Franklin (2006), así como otros autores, han inferido que la decisión del voto y la campaña electoral en sí en este tipo de comicios se ha basado en la agenda nacional ya que, se argumenta, los temas europeos han tendido siempre a estar relegados a un segundo plano, si es que aparecen durante la campaña electoral. Este fenómeno, además, se ha sostenido en el tiempo (véase Schmitt y Teperoglou, 2015; Hix y Marsh, 2011).

\subsection{ELECCIONES AL PARLAMENTO EUROPEO}

Aun siendo entendidas como elecciones de segundo orden, debemos ir más allá del marco teórico de ese tipo de elecciones y buscar las particularidades de las campañas electorales al Parlamento Europeo, objetivo básico de este artículo. Ya en la citada obra de Reif y Schmitt de 1980, estos autores apuntan al hecho de que las elecciones al Parlamento Europeo son más nacionales que europeas. Este punto fue corroborado por Franklin (2006), quien analizó las diferentes elecciones al Parlamento Europeo que tuvieron lugar entre 1979 y 2004 . Entre sus conclusiones, este autor señala que en muy pocas ocasiones el contenido de las propuestas de los partidos políticos forma parte de ninguna agenda mediática europea.

Este enfoque nacional que hacen los partidos se traslada también en la decisión de voto por parte de los ciudadanos. Gracias a los datos y encuestas postelectorales, sabemos que los ciudadanos de la Unión Europea que acudieron a las urnas en las elecciones de 2009 tomaron la decisión de su voto basados en temas de agenda nacional, ya que los 
temas europeos habían brillado por su ausencia durante la campaña electoral (Schmitt y Teperoglou, 2015).

En este sentido, autores como Norris y Reif $(1997$, p. 110) no dudan en calificar la campaña electoral al Parlamento Europeo como "decepcionante", al mismo tiempo que señalan como decepción el hecho de que los ciudadanos, desde su punto de vista, "fallen" a la hora de interesarse por los comicios al Parlamento Europeo, que, convocatoria tras convocatoria, han ido menguando en participación (Norris y Reif, 1997). Para los ciudadanos, apuntan algunos estudios, las elecciones al Parlamento Europeo no son más que referéndums sobre qué tan bien lo está haciendo el partido que está en el gobierno de sus respectivos países (Franklin, 2006).

Cierto es que los partidos en el gobierno tienden a la pérdida de votos en las elecciones al Parlamento Europeo, pero no menos cierto es que algunos autores señalan la entrada, en 2004, de 10 nuevos Estados miembro a la UE, procedentes del centro y Este de Europa como causante de un voto protesta contra la integración europea (apoyada por los partidos mayoritarios) (Van der Eijk y Van Egmond, 2007). Sea como fuere, las últimas dos convocatorias electorales al Parlamento Europeo, en 2014 y 2019, se han caracterizado por un incremento de apoyo a partidos extremistas y partidos llamados eurófobos, contrarios a la integración en la Unión Europea (Schmitt y Teperoglou, 2017). A pesar de todo, los grupos parlamentarios mayoritarios, el Grupo Popular Europeo y el Grupo Socialdemócrata, conservan la mayoría de los eurodiputados.

\section{METODOLOGÍA}

Esta investigación parte de una metodología cuantitativa. En concreto, se centra en el análisis de las campañas electorales de los diferentes partidos políticos y coaliciones de España y Francia que, al menos en una ocasión entre las elecciones de 2004 y 2019, han obtenido representación al Parlamento Europeo.

A tal efecto, se ha realizado un análisis de contenido de todas las informaciones al respecto de las elecciones al Parlamento Europeo aparecidas durante los 15 días previos a cada una de las elecciones tanto en El País como en Le Monde. La elección de estos 
diarios se ha hecho atendiendo al nivel de fiabilidad y a la importancia que tienen en España y Francia respectivamente, como acredita el informe anual Digital News Report, del Reuters Institute, que los sitúa como diarios de referencia de la información para sus ciudadanos. Además, El País ha sido en el periodo entre 2004 y 2019 el diario que más ha liderado los índices de difusión OJD y EGM, mientras que Le Monde ha hecho lo mismo entre los medios auditados por OJD ACPM. A dicha justificación se le suma un factor histórico, el de que, entre los medios más seguidos y usados para informarse por los ciudadanos de cada uno de los países, El País y Le Monde son de los escasos que se publicaban ya antes de 2004, año en el que empieza el presente estudio (y que se han mantenido hasta la actualidad), dejando a un lado aquellos medios que la literatura califica como tabloides (Filas y Pnatea, 2009)

Para garantizar que este análisis de contenido cumple con las características propias descritas por la literatura (objetividad, sistematicidad y capacidad de generalización), se ha seguido el procedimiento que describen la mayoría de los textos que presentan el análisis de contenidos como una de las metodologías de uso frecuente en las ciencias sociales (Andreu, 2000).

El corpus de nuestro análisis de contenido es de un total de 688 piezas periodísticas publicadas en uno de los dos diarios mencionados durante todas las campañas electorales al Parlamento Europeo entre las elecciones de 2004 y las de 2019. En total se han analizado las elecciones de 2004, 2009, 2014 y 2019. Así, las unidades de registro se han codificado en unidades de objeto, de personaje (al analizar quién protagoniza cada pieza informativa), de medición de tiempo y espacio y de análisis de la propia pieza informativa. Por consiguiente, aquí se muestra las diferentes variables y codificación de análisis para cumplir con las características de exclusividad, exhaustividad y homogeneidad que recomienda la literatura (Victoria, 2002). El volcado de los datos y el trabajo estadístico se ha realizado con la herramienta SPSS 22.0 y las variables que se han tenido en cuenta son las siguientes:
A) País al cual pertenece el partido analizado
B) Año de las elecciones al Parlamento Europeo 
C) Grupo político del Parlamento Europeo al cual pertenece el partido

D) ¿Estaba dicho partido previamente antes en el Parlamento Europeo?

E) Ámbito del tema que se trata en la información

F) Temática tratada en la información

G) Ámbito del protagonista de la información

H) Género del protagonista de la información

I) Edad del protagonista de la información

J) Ocupación del protagonista de la información

K) Tipo de pieza periodística

Además de la presentación de resultados general, cada una de estas variables nos ayudará a comprender con más detalle las características de la comunicación política en la política europea. Así, mientras la variable A nos permitirá inferir posibles diferencias entre países, la B nos ayudará de manera similar para inferir posible evolución a lo largo de los años.

Por lo que respecta a la variable C, la de los grupos políticos, su codificación se ha realizado conforme al final de la última legislatura finalizada antes de la presentación de este artículo (2014-2019). Esto se debe al hecho del cambio de nomenclatura y también de composición de los grupos políticos. Se ha clasificado a cada partido en el grupo político al que pertenecía o al cual se unió al celebrarse los comicios de ese año. Es decir: si un partido pertenecía en 2004 al Partido Popular Europeo (PPE) pero en 2014 decidió pasar al grupo de los Conservadores Europeos (ECR), en el análisis de 2004 se le asimiló en el PPE y en 2014 en el ECR.

Para los diferentes cambios de nomenclatura y desapariciones de grupos políticos, se ha asimilado a los partidos que pertenecían a un determinado grupo político al 'heredero', entendiendo heredero como aquel grupo político de nueva nomenclatura que aglutina a la gran mayoría de partidos políticos que aglutinaba el anterior grupo político.

\section{RESULTADOS}

\subsection{RESULTADOS}


En esta sección se resumen los resultados más importantes del análisis cuantitativo, siendo dichos resultados completos más extensos. El que más destaca es el que se observa en la Tabla 1, donde se confirma que la campaña electoral de las elecciones al Parlamento Europeo se caracteriza por una agenda temática basada en temas noeuropeos. Los temas considerados de ámbito simplemente europeos son el 35,9\% de una campaña electoral que, precisamente, acabará en la elección de eurodiputados para el Parlamento Europeo. Una característica observada es como, en no pocas ocasiones, los líderes y partidos políticos usan temáticas transversales, que se podrían considerar europeas, para añadirle el punto de vista del conflicto nacional o regional y así convertirlo en un tema de interés para sus votantes.

Tabla 1. Ámbito del tema tratado durante la campaña

\begin{tabular}{|c|c|}
\hline Ámbito del tema & Porcentaje de intervenciones \\
\hline Regional & $2,3 \%$ \\
\hline Estatal & $29,4 \%$ \\
\hline Europeo & $35,9 \%$ \\
\hline Regional / Estatal & $4,4 \%$ \\
\hline Regional / Europeo & $2,5 \%$ \\
\hline Estatal / Europeo & $25,6 \%$ \\
\hline
\end{tabular}

Fuente: Elaboración propia

A este respecto, no se observan tampoco grandes diferencias en cuanto al ámbito del tema tratado ni por países ni por evolución histórica. En la Tabla 2 se pueden observar cómo los valores son bastante parejos entre Francia y España, a excepción de una tendencia más centralista en Francia. Por su parte, respecto a la evolución a lo largo del tiempo, vemos una tendencia nacida en 2014 (y confirmada en 2019) por la apuesta por la agenda europea. Aunque aún no supone ni la mitad del tiempo dedicado durante la campaña, los temas de afectación exclusivamente europeos se van imponiendo a lo largo que nos acercamos a las elecciones europeas más recientes. 
Tabla 2. Ámbito del tema tratado durante la campaña según el país y el año de las elecciones

\begin{tabular}{|c|c|c|c|}
\hline $\begin{array}{c}\text { Ámbito } \\
\text { del tema }\end{array}$ & $\begin{array}{c}\text { Porcentaje } \\
\text { general }\end{array}$ & España & Francia \\
\hline Regional & $2,3 \%$ & $3,9 \%$ & $0,3 \%$ \\
\hline Estatal & $29,4 \%$ & $30,1 \%$ & $28,4 \%$ \\
\hline Europeo & $35,9 \%$ & $34,6 \%$ & $37,6 \%$ \\
\hline $\begin{array}{c}\text { Regional / } \\
\text { Estatal }\end{array}$ & $4,4 \%$ & $7,3 \%$ & $0,7 \%$ \\
\hline $\begin{array}{c}\text { Regional / } \\
\text { Europeo }\end{array}$ & $2,5 \%$ & $3,1 \%$ & $1,6 \%$ \\
\hline $\begin{array}{c}\text { Estatal / } \\
\text { Europeo }\end{array}$ & $25,6 \%$ & $20,9 \%$ & $31,4 \%$ \\
\hline
\end{tabular}

\begin{tabular}{|c|c|c|c|}
\hline $\mathbf{2 0 0 4}$ & $\mathbf{2 0 0 9}$ & $\mathbf{2 0 1 4}$ & $\mathbf{2 0 1 9}$ \\
\hline $2,8 \%$ & $2,8 \%$ & $1,8 \%$ & $2,3 \%$ \\
\hline $34,6 \%$ & $40,4 \%$ & $35,4 \%$ & $20,5 \%$ \\
\hline $23,4 \%$ & $19,3 \%$ & $31,7 \%$ & $48,4 \%$ \\
\hline $4,7 \%$ & $12,8 \%$ & $4,9 \%$ & $1 \%$ \\
\hline $3,7 \%$ & 2,8 & $1,8 \%$ & $2,3 \%$ \\
\hline $30,8 \%$ & $22 \%$ & $24 \%$ & $25,6 \%$ \\
\hline
\end{tabular}

Fuente: Elaboración propia

En un sentido parecido, se observa cómo, en líneas generales, no solo la temática se aleja de la agenda europea, sino que los propios protagonistas de la campaña también. En la Tabla 3 se observa como en las elecciones al Parlamento Europeo hay una fuerte presencia de líderes nacionales (entiéndase como o bien presidentes, primeros ministros, jefes de la oposición a nivel nacional u otros líderes sin responsabilidades ni candidatura a nivel europeo). Aunque el lector pueda observar una divergencia en las elecciones de 2019, esto se debe al hecho de que en España coincidieron el mismo día comicios europeos con elecciones municipales en toda España y regionales en algunas Comunidades Autónomas, relegando el contenido sobre las elecciones al Parlamento Europeo a piezas de análisis y opinión, muchas de ellas escritas por líderes de opinión y académicos.

Tabla 3. Ámbito de acción del protagonista de la acción de campaña analizada

\begin{tabular}{|c|c|c|c|}
\hline $\begin{array}{c}\text { Ámbito de } \\
\text { acción }\end{array}$ & $\begin{array}{c}\text { Porcentaje } \\
\text { general }\end{array}$ & España & Francia \\
\hline Estatal & $34,9 \%$ & $32,2 \%$ & $38,9 \%$ \\
\hline Europeo & $40 \%$ & $50,5 \%$ & $24,1 \%$ \\
\hline Ambos $^{2}$ & $7,5 \%$ & $2,1 \%$ & $15,7 \%$ \\
\hline $\begin{array}{c}\text { Líder } \\
\text { opinión } \\
\text { social }\end{array}$ & $17,6 \%$ & $15,2 \%$ & $21,3 \%$ \\
\hline
\end{tabular}

\begin{tabular}{|c|c|c|c|}
\hline $\mathbf{2 0 0 4}$ & $\mathbf{2 0 0 9}$ & $\mathbf{2 0 1 4}$ & $\mathbf{2 0 1 9}$ \\
\hline $42,5 \%$ & $56,6 \%$ & $25 \%$ & $26 \%$ \\
\hline $44,3 \%$ & $35,8 \%$ & $60,6 \%$ & $20,8 \%$ \\
\hline $7,5 \%$ & $1,9 \%$ & $4,4 \%$ & $13,9 \%$ \\
\hline $5,7 \%$ & $5,7 \%$ & $10 \%$ & $39,3 \%$ \\
\hline
\end{tabular}

Fuente: Elaboración propia

\footnotetext{
2 En "Ambos" se entienden aquellos candidatos o personajes con cargo político relacionado con la Unión Europea pero con anteriores responsabilidades nacionales o lo contrario (actualmente con responsabilidad a nivel nacional pero ex eurodiputado, por ejemplo).

${ }^{3}$ Se incluye toda persona sin responsabilidad política ni partidista en el momento de su intervención en campaña o la publicación de un artículo de opinión u análisis.
}

RAE-IC, Revista de la Asociación Española de Investigación de la Comunicación vol. 8, núm. 16 (2021), 313-336 
Otro aspecto donde podemos inferir cierta evolución es en los dos datos demográficos básicos: la edad y el género de quien protagoniza cada acto de campaña o intervención en medios de comunicación. En ambos casos observamos muy pocas diferencias entre países, pero sí podemos inferir diferencias a lo largo de las cuatro elecciones analizadas.

En el primer caso, el de la edad, y con la excepción de los comicios de 2014, se observa cómo se ha intentado revertir el alto porcentaje de campaña electoral protagonizada por lo que hemos llamado 'veteranos', aquellas personas de más de 50 años (en el momento de su intervención en la campaña). Dicha tendencia se ha acentuado en las elecciones de 2019 y queda por confirmar (o no) con las futuras elecciones al Parlamento Europeo de 2024.

Tabla 4. Edad del protagonista de cada intervención de campaña electoral

\begin{tabular}{|c|c|c|c|}
\hline Edad & $\begin{array}{c}\text { Porcentaje } \\
\text { general }\end{array}$ & España & Francia \\
\hline $\begin{array}{c}\text { Joven (18- } \\
34)\end{array}$ & $3,7 \%$ & $2,4 \%$ & $5,7 \%$ \\
\hline $\begin{array}{c}\text { Consolidado } \\
\text { (35-49) }\end{array}$ & $28,1 \%$ & $27,1 \%$ & $29,7 \%$ \\
\hline $\begin{array}{c}\text { Veterano } \\
\text { (50 y más) }\end{array}$ & $68,2 \%$ & $70,5 \%$ & $64,6 \%$ \\
\hline
\end{tabular}

\begin{tabular}{|c|c|c|c|}
\hline $\mathbf{2 0 0 4}$ & $\mathbf{2 0 0 9}$ & $\mathbf{2 0 1 4}$ & $\mathbf{2 0 1 9}$ \\
\hline $3 \%$ & $0,9 \%$ & $1,9 \%$ & $7,6 \%$ \\
\hline $31,7 \%$ & $36,1 \%$ & $9,4 \%$ & $38,2 \%$ \\
\hline $65,3 \%$ & $63 \%$ & $88,7 \%$ & $54,1 \%$ \\
\hline
\end{tabular}

Fuente: Elaboración propia (se han excluido editoriales y artículos de opinión escritos por más de un autor)

Precisamente en el caso de la edad es donde se observan más diferencias entre los distintos grupos políticos, siendo observable en la Tabla 5 como los grupos de izquierda apuesta, no diremos por la juventud, pero sí más porque su campaña electoral la protagonicen candidatos y líderes de mediana edad. 
Tabla 5. Edad del protagonista de cada intervención de campaña electoral según el grupo político europeo al cual pertenece su partido

\begin{tabular}{|c|c|c|c|c|c|c|c|c|c|c|}
\hline Edad & $\begin{array}{l}\text { Porcentaje } \\
\text { general }\end{array}$ & Grupo Popular & $\begin{array}{c}\text { Grupo } \\
\text { Socialdemócrata }\end{array}$ & $\begin{array}{l}\text { Grupo } \\
\text { Conservadores } \\
\text { y Reformistas }\end{array}$ & $\begin{array}{c}\text { ALDE - } \\
\text { Demócratas y } \\
\text { Liberales }\end{array}$ & $\begin{array}{l}\text { Izquierda } \\
\text { Unitaria - } \\
\text { Verde }\end{array}$ & Verdes / ALE & $\begin{array}{l}\text { EFDD (Libertad } \\
\text { Democracia } \\
\text { Directa) }\end{array}$ & $\begin{array}{l}\text { Europa de } \\
\text { Naciones y } \\
\text { Libertades }\end{array}$ & No Inscritos \\
\hline Joven (18-34) & $3,7 \%$ & $2,5 \%$ & $3,4 \%$ & $0 \%$ & $0 \%$ & $8,8 \%$ & $8 \%$ & $0 \%$ & $0 \%$ & $0 \%$ \\
\hline $\begin{array}{c}\text { Consolidado } \\
\text { (35-49) }\end{array}$ & $28,1 \%$ & $14,3 \%$ & $30,5 \%$ & $25 \%$ & $27,3 \%$ & $19,3 \%$ & $40 \%$ & $0 \%$ & $50 \%$ & $0 \%$ \\
\hline $\begin{array}{c}\text { Veterano (50 } \\
\text { y más) }\end{array}$ & $68,2 \%$ & $83,2 \%$ & $66,1 \%$ & $75 \%$ & $72,7 \%$ & $71,9 \%$ & $52 \%$ & $100 \%$ & $50 \%$ & $100 \%$ \\
\hline
\end{tabular}

Fuente: Elaboración propia (se han excluido editoriales y artículos de opinión escritos por más de un autor) 
Tabla 6. Temática de cada acto de campaña según el grupo político al cual pertenece el partido político

\begin{tabular}{|c|c|c|c|c|c|c|c|c|c|c|}
\hline Temática & $\begin{array}{l}\text { Porcentaje } \\
\text { general }\end{array}$ & Grupo Popular & $\begin{array}{c}\text { Grupo } \\
\text { Socialdemócrata }\end{array}$ & $\begin{array}{c}\text { Grupo } \\
\text { Conservadores } \\
\text { y Reformistas }\end{array}$ & $\begin{array}{c}\text { ALDE - } \\
\text { Demócratas y } \\
\text { Liberales }\end{array}$ & $\begin{array}{l}\text { Izquierda } \\
\text { Unitaria - } \\
\text { Verde }\end{array}$ & Verdes / ALE & $\begin{array}{l}\text { EFDD (Libertad } \\
\text { Democracia } \\
\text { Directa) }\end{array}$ & $\begin{array}{l}\text { Europa de } \\
\text { Naciones y } \\
\text { Libertades }\end{array}$ & No Inscritos \\
\hline Agricultura & $0,9 \%$ & $0,8 \%$ & $0,8 \%$ & $0 \%$ & $0 \%$ & $0 \%$ & $0 \%$ & $0 \%$ & $0 \%$ & $0 \%$ \\
\hline Política & $63,5 \%$ & $63,6 \%$ & $65,3 \%$ & $75 \%$ & $61,4 \%$ & $56,5 \%$ & $61,3 \%$ & $100 \%$ & $73,1 \%$ & $75 \%$ \\
\hline Economía & $16 \%$ & $19,8 \%$ & $20,7 \%$ & $0 \%$ & $16,9 \%$ & $24,2 \%$ & $9,7 \%$ & $0 \%$ & $0 \%$ & $0 \%$ \\
\hline $\begin{array}{l}\text { Asuntos } \\
\text { sociales y } \\
\text { justicia }\end{array}$ & $5,2 \%$ & $4,1 \%$ & $3,3 \%$ & $0 \%$ & $8,4 \%$ & $6,5 \%$ & $3,2 \%$ & $0 \%$ & $3,8 \%$ & $0 \%$ \\
\hline $\begin{array}{l}\text { Seguridad / } \\
\text { Terrorismo }\end{array}$ & $1,5 \%$ & $1,7 \%$ & $3,3 \%$ & $0 \%$ & $2,4 \%$ & $1,6 \%$ & $0 \%$ & $0 \%$ & $0 \%$ & $0 \%$ \\
\hline Geopolítica & $6,4 \%$ & $6,6 \%$ & $4,1 \%$ & $0,0 \%$ & $4,8 \%$ & $3,2 \%$ & $0 \%$ & $0 \%$ & $11,5 \%$ & $25 \%$ \\
\hline $\begin{array}{c}\text { Medio } \\
\text { ambiente }\end{array}$ & $3,3 \%$ & $0,8 \%$ & $1,7 \%$ & $0 \%$ & $3,6 \%$ & $4,8 \%$ & $19,4 \%$ & $0 \%$ & $0 \%$ & $0 \%$ \\
\hline Transporte & $0,6 \%$ & $0 \%$ & $0 \%$ & $0 \%$ & $0 \%$ & $0 \%$ & $6,5 \%$ & $0 \%$ & $0 \%$ & $0 \%$ \\
\hline $\begin{array}{l}\text { Inmigración } \\
\text { y vecindad }\end{array}$ & $2,6 \%$ & $2,4 \%$ & $0,8 \%$ & $25 \%$ & $2,4 \%$ & $3,2 \%$ & $0 \%$ & $0 \%$ & $11,5 \%$ & $0 \%$ \\
\hline
\end{tabular}

Fuente: Elaboración propia 
Y si se trata de comentar diferencias en cuanto a los diferentes grupos políticos, otra de las principales la encontramos en los temas de campaña. Si observamos la Tabla 6, en líneas generales la principal temática escogida por todos es la Política, todos aquellos temas relacionados con propuestas políticas, debates generales, ataques directos a otros partidos políticos o a la acción de gobierno, etc. En segundo lugar, destaca la apuesta por los temas económicos. Y a partir de aquí, las divergencias. Mientras los dos principales grupos del Parlamento Europeo, Populares y Socialdemócratas, se centran en estas dos temáticas, otros grupos minoritarios, especialmente de izquierda, apuestan por temáticas que la ciencia política de manera continua les ha atribuido, como el medio ambiente. Por su parte, temáticas como la de inmigración destaca en el discurso de grupos como el de Conservadores y Reformistas y el de la Europa de las Naciones y Libertades.

En el caso del género, existe una brecha enorme entre hombres y mujeres, siendo claramente los varones los protagonistas de las campañas electorales al Parlamento Europeo. Como se observa en la Tabla 7, esta brecha se ha ido reduciendo, especialmente en 2014 y 2019 gracias al protagonismo en Francia de Marine Le Pen y en España de la que fuese cabeza de lista por el PSOE, Elena Valenciano, aunque queda por ver si esta reducción de la brecha continúa en las elecciones de 2024.

Tabla 7. Género del protagonista de cada intervención de campaña electoral

\begin{tabular}{|c|c|c|c|}
\hline Género & $\begin{array}{c}\text { Porcentaje } \\
\text { general }\end{array}$ & España & Francia \\
\hline Hombre & $76,4 \%$ & $79 \%$ & $72,4 \%$ \\
\hline Mujer & $23,6 \%$ & $21 \%$ & $27,6 \%$ \\
\hline
\end{tabular}

\begin{tabular}{|c|c|c|c|}
\hline $\mathbf{2 0 0 4}$ & $\mathbf{2 0 0 9}$ & $\mathbf{2 0 1 4}$ & $\mathbf{2 0 1 9}$ \\
\hline $95,1 \%$ & $84,3 \%$ & $70,4 \%$ & $66,1 \%$ \\
\hline $4,9 \%$ & $15,7 \%$ & $29,6 \%$ & $33,9 \%$ \\
\hline
\end{tabular}

Fuente: Elaboración propia (se han excluido editoriales y artículos de opinión escritos por más de un autor)

\subsection{DISCUSIÓN}

Aunque la campaña electoral al Parlamento Europeo presenta las características teóricas propuestas por Maarek (2011) y Hix y Marsh (2011), queda claro que, si analizamos el contenido y a los protagonistas de dichas campañas, se dibujan ciertas particularidades. 
La principal, descrita ya con anterioridad por otros autores, es que la agenda que debería ser predominante -en este caso la europea- queda en un segundo plano. Con el análisis del presente artículo podemos poner cifras a las propuestas teóricas de Reif y Schmitt (1980) y otros autores comentados en la sección del marco teórico.

El hecho de que solo el 35,9\% de los temas y las intervenciones de campaña versen únicamente de temas de afectación europea expone cómo los partidos políticos, de ámbito nacional por las características propias de los comicios al Parlamento Europeo, se toman estas elecciones como un examen: en el caso del gobierno como una reválida a su gestión y, en el caso de los partidos en la oposición, como un sondeo sobre la percepción del público. Prueba de ello es el hecho de que tan solo el $40 \%$ de las intervenciones de campaña están protagonizadas por un candidato al Parlamento Europeo o un eurodiputado, siendo el $34,9 \%$ de los actos protagonizados por actores políticos de ámbito estatal (como el líder del partido, esté en el gobierno o no).

Por su parte, los datos confirman que, con ligeras variaciones entre países y entre los diferentes comicios analizados, el Parlamento Europeo se ha caracterizado por ser lo que tanto informalmente como algunos expertos han calificado como "cementerio de elefantes", donde predominan los diputados de más de 50 años que están ante sus últimos años en la política activa (Zabaltza, 2009). Esta es ya una de las características claras de la campaña a las elecciones al Parlamento Europeo: el uso de actores político hacia el final de sus carreras, en tanto que, en la línea de lo comentado por Reif y Schmitt (1980) y Norris y Reif (1997), para los partidos políticos nacionales, las elecciones al Parlamento Europeo no tienen un peso tan específico como las elecciones generales nacionales. A esto puede contribuir la propia configuración de los comicios, que, como señalaba Morata (1998), se basa en listas electorales nacionales y circunscripción electoral por Estado, en vez de una circunscripción única que englobase a todos los Estados miembro en cada ocasión.

En cuanto al género, nos encontramos en lo que algunos autores denominan un contexto de "feminización de la política" (Ochoa, 2021), más en la comunicación de campaña en las elecciones al Parlamento Europeo la brecha entre el protagonismo de 
los hombres y las mujeres sigue siendo muy llamativa, siendo cada vez más pequeña, pero quedándose en las elecciones de 2019 en un 66\% de presencia masculina.

Por lo que respecta a las temáticas, más allá de su carácter nacional o europeo, destaca el hecho de que los partidos que pertenecen a los grupos parlamentarios más modestos apuestan en mayor medida por temas específicos, más allá de la discusión política tradicional y la batalla dialéctica. Medio ambiente y asuntos sociales (en el caso de la izquierda) e inmigración y geopolítica (en el caso de la derecha y los partidos euroescépticos) aparecen mucho más en campañas electorales de los partidos pequeños, de nuevo en la línea de lo expresado por Norris y Reif (1997) pero también persiguiendo el objetivo subrayado por Hobolt et al. (2009): desmarcarse de los partidos en el gobierno y ganar apoyo frente a ellos en unas elecciones de segundo orden donde no se observa con igual intensidad el 'voto útil' como factor condicionante del voto.

\section{CONCLUSIONES}

Tras analizar de manera cuantitativa diferentes factores propios de la estrategia electoral y comunicación relativa a las elecciones al Parlamento Europeo, este texto debe servir para reforzar la idea de dichas elecciones como elecciones de segundo grado, al quedar probado que en las elecciones de 2019, las más recientes celebradas a fecha de entrega de este artículo, la agenda europea seguía siendo secundaria en las campañas electorales de aquellos partidos que obtuvieron representación en el Parlamento Europeo.

En este sentido cabe destacar también el hecho de que los propios candidatos en las elecciones al Parlamento Europeo no cogen el protagonismo que se espera si se compara con otro tipo de elecciones, como las elecciones nacionales e incluso las regionales. Además, comparando dos países como España y Francia queda probado que esta característica ya mencionada en 1980 por Reif y Schmitt es válida sea donde sea que se celebren los comicios al Parlamento Europeo.

Si hablamos de la evolución temporal, será interesante para futuras investigaciones comprobar si las elecciones al Parlamento Europeo de 2024 acaban de desafiar a Reif y 
Schmitt en tanto la agenda europea toma el control en la campaña electoral o continúa siendo secundaria, así como el uso de actores políticos de ámbito europeo o nacional. Especialmente relevante es esta cuestión en el caso español, donde el contenido por las elecciones al Parlamento Europeo en los medios se vio superado por coincidir en fecha con elecciones locales y regionales.

Resueltas algunas de las cuestiones relacionadas con las características que se les atribuyen históricamente a las elecciones al Parlamento Europeo en tanto que elecciones de segundo grado, el reto de futuras investigaciones está en confirmar otras de las características, como la del nivel de voto nulo y el efecto sobre partidos en el gobierno y partidos pequeños. En los datos presentados, algo que queda claro es que esos grupos políticos más pequeños apuestan por temas de campaña más arriesgados, saliendo de la política general para tratar otros como la migración, la geopolítica o el medio ambiente. A todo eso, faltará añadirle el componente del éxito electoral para inferir sobre la efectividad de una u otra estrategia de comunicación en campaña electoral al Parlamento Europeo.

\section{REFERENCIAS BIBLIOGRÁFICAS}

Aira, T. (2008). Màrqueting polític: l'art de guanyar eleccions. Barcelona: Trípodos.

Andreu, J. J. (2000). Las técnicas de análisis de contenido: una revisión actualizada. Fundación Centro Estudios Andaluces, 10(2), 1-34.

Auel, K. y Rittberger, B. (2006). Fluctuant nec merguntur: the European Parliament, national parliaments, and European integration. En J. Richardson, European Union: power and policy-making (pp. 121-146). Oxor: Routledge.

Bennet, W. e lyengar, S. (2008). A new era of minimal effects? The changing foundations of political communication. Journal of Communication, 58, 707-731.

Canel, M. (2006). Comunicación Política. Una guía para su estudio y práctica. Madrid: Tecnos 
Castro, L. (2012). El marketing político en Estados Unidos: el caso Obama. Norteamérica, 7(1), 209-222.

Denton, R. y Woodward, G. (1998). Political Communication in America. Londres: Praeger.

Filas, R. y Pnatea, P. (2009). Media in Poland and Public Discourse. En A. Czepek, M. Hellwig y E. Nowak, Press freedom and pluralism in Europe (pp. 141-163). Bristol: Intellect

Frankin, M. (2006). European elections and the European voter. En J. Richardson, European Union: power and policy-making (pp. 227-246). Oxon: Routledge.

Hix, S. y Marsh, M. (2007). Punishment or protest? Understanding European Parliament elections. The Journal of Politics, 69(2), 495-510.

Hix, S. y Marsh, M. (2011). Second-order effects plus pan-European political swings: an analysis of European Parliament elections across time. Electoral Studies, 30, 4-15.

Hobolt, S.; Spoon, K. y Tilley, J. (2009). A vote against Europe? Explaining defection at the 1999 and 2004 European Parliament elections. British Journal of Political Science, 39(1), 93-115.

López-García, G., Llorca-Abad, G., Valera-Ordaz, L. y Peris-Blanes, A. (2018). Los debates electorales, ¿el último reducto frente la mediatización? Un estudio de caso de las elecciones generales españolas de 2015. Palabra Clave, 21(3), 772-797.

Maarek, P. (2011). Campaign communication \& Political Marketing. Oxford: WileyBlackwell

Martínez Pandiani, G. (2004). Marketing Político. Buenos Aires: Urgeman Editor.

Mazzoleni, G. (2010). La Comunicación Política. Madrid: Alianza.

Morata, F. (1998). La Unión Europea: procesos, actores y políticas. Barcelona: Ariel. 
Norris, P. y Reif, K. (1997). Second Order Elections revisited. European Journal of Political Research, 31, 109-124.

Ochoa, E. (2021). Feminización de la política. Pluralidad y consenso, 47, 32-35.

Olmo López, A. y Navarro Moreno, J. A. (2014). Estrategias de comunicacion de la Unión Europea: experiencias para el fomento de la información. En Primer Congreso Internacional Infoxicación: mercado de la información y psique, Libro de Actas (pp. 495517). Ladecom, Facultad de Comunicación, Universidad de Sevilla.

Reif, K. y Schmitt, H. (1980). Nine second-order national elections - A conceptual framework for the analysis of European election results. European Journal of Political Research, 8, 3-44.

Reuters Institute (2021). Digital News Report 2021. Oxford: University of Oxford Schmitt, H. y Teperoglou, E. (2015). The 2014 European Parliament elections in Southern Europe: second-order or critical elections?. South European Society and Politics, 20(3), 287-309. https://doi.org/10.1080/13608746.2015.1078271

Schmitt, H. y Teperoglou, E. (2017). The study of less important elections. En K. Arzheimer, J. Evans y M. Lewis-Beck, The SAFE Handbook of Electoral Behaviour (pp. 56-71). Londres: SAGE.

Van der Eijk, C. y Van Egmond, M. (2007). Political effects of low turnout in national and European Elections. Electoral Studies, 26(3), 561-573.

Victoria, J. (2002). El análisis de contenido: una técnica para explorar y sistematizar información. XXI Revista de Educación, 4, 95-105.

Zabaltza, X. (30 de mayo de 2009). Por una lengua común europea. El País. Recuperado de https://elpais.com/diario/2009/05/30/opinion/1243634404_850215.html 